\title{
Tumor-associated macrophages in
}

\section{cancer-related inflammation}

\author{
Alberto Mantovani \\ Istituto Clinico Humanitas IRCCS, \\ University of Milan, Italy \\ alberto.mantovani@ \\ humantasresearch.it
}

\section{Financial \& competing} interests disclosure

The author has no relevant affiliations or financial involvement with any organization or entity with a financial interest in or financial conflict with the subject matter or materials discussed in the manuscript. This includes employment, consultancies, honoraria, stock ownership or options, expert testimony, grants or patents received or pending, or royalties.

No writing assistance was utilized in the production of this manuscript.
As early as the 19th Century, it was perceived that cancer is linked to inflammation. This perception has waned for a long time; however, recent years have seen a renaissance of the inflammation-cancer connection stemming from different lines of work and leading to a generally accepted paradigm [1-4]. Recent efforts have shed new light on molecular and cellular pathways linking inflammation and cancer [4]. Two pathways link inflammation and cancer. In the intrinsic pathway, activation of different classes of oncogenes drives the expression of inflammationrelated programs, which guide the construction of an inflammatory microenvironment. In the extrinsic pathway, inflammatory conditions promote cancer development (e.g., colitis-associated cancer of the intestine). Key orchestrators at the intersection of the intrinsic and extrinsic pathway include transcription factors (e.g., nuclear factor $\kappa B$ ) [5], cytokines (e.g., TNF) and chemokines. Thus, inflammation is a key component of the tumor microenvironment and a target for pharmacologic intervention [6]. In a seminal contribution, Hanahan and Weinberg identified six hallmarks of cancer [7]. We have surmized that cancerrelated information represents a seventh hallmark of cancer [8]. Macrophages are key orchestrators of chronic inflammation. They respond to microenvironmental signals with polarized genetic and functional programs. M1 macrophages, which are classically activated by microbial products and IFN- $\gamma$, are potent effector cells that kill microorganisms and tumors. By contrast, $\mathrm{M} 2$ cells tune inflammation and adaptive immunity, promote cell proliferation by producing growth factors and products of the arginase pathway (ornithine and polyamines), scavenge debris by expressing scavenger receptors, and promote angiogenesis, tissue remodeling and repair. M1 and M2 cells represent simplified extremes of a continuum of functional states. Available information suggests that tumor-associated macrophages are a prototypic M2 population. M2 polarization of phagocytes sets these cells in a tissue remodeling and repair mode and orchestrates the smoldering and polarized chronic inflammation associated with established neoplasia. Recent studies have begun to address the central issue of the relationship between genetic events causing cancer and activation of protumor inflammatory reactions. Rearrangement of the RET oncogene (RETIPTC) is a frequent, causative and sufficient event in papillary carcinoma of the thyroid. It was recently observed that RET/PTC activates a proinflammatory genetic program in primary human thyrocytes, including, in particular, chemokines and chemokine receptors. These molecules are also expressed in vivo and more so in metastatic tumors. These results highlight a direct connection between an early, causative and sufficient oncogene rearrangement and activation of a proinflammatory program in a human tumor. Therapeutic targeting of cancer-promoting inflammatory reactions is in its infancy, and its development is crucially dependent on defining the 
underlying cellular and molecular mechanisms in relevant systems. Chemokines are prime targets for interfering with tumor promotion by inflammatory reactions. Ongoing efforts along this line are encouraging. Inflammatory mediators can promote invasion and metastasis. In particular, expression of chemokine receptors and production of cognate ligands underlies homing of tumor cells at different anatomical sites $[9,10]$.

\section{Bibliography}

1 Balkwill F, Mantovani A: Inflammation and cancer: back to Virchow? Lancet 357, 539-545 (2001).

2 Coussens LM, Werb Z: Inflammation and cancer. Nature 420, 860-867 (2002).

3 Balkwill F, Charles KA, Mantovani A: Smoldering and polarized inflammation in the initiation and promotion of malignant disease. Cancer Cell 7, 211-217 (2005).

4 Mantovani A, Allavena P, Sica A, Balkwill F: Cancer-related inflammation. Nature 454, 436-444 (2008).
5 Rius J, Guma M, Schachtrup C et al:: NF- $\mathrm{B}$ links innate immunity to the hypoxic response through transcriptional regulation of HIF-1 $\alpha$. Nature 453, 807-811 (2008).

6 Harrison ML, Obermueller E, Maisey NR et al.: Tumor necrosis factor $\alpha$ as a new target for renal cell carcinoma: two sequential Phase II trials of infliximab at standard and high dose. J. Clin. Oncol. 25, 4542-4549 (2007).

7 Hanahan D, Weinberg RA: Hallmarks of cancer: the next generation. Cell 144, 646-674 (2011).
8 Mantovani A: Cancer: inflaming metastasis. Nature 457, 36-37 (2009).

9 Mantovani A, Savino B, Locati M, Zammataro L, Allavena P, Bonecchi R: The chemokine system in cancer biology and therapy. Cytokine Growth Factor Rev. 21, 27-39 (2010).

10 Marchesi F, Piemonti L, Mantovani A, Allavena P: Molecular mechanisms of perineural invasion, a forgotten pathway of dissemination and metastasis. Cytokine Growth Factor Rev. 21, 77-82 (2010). 